\title{
Influencia de la obesidad en los costos en salud y en el ausentismo laboral de causa médica en una cohorte de trabajadores
}

\author{
Aldo Zárate ${ }^{1}$, Marco Crestto, Alberto Maiz ${ }^{1}$, G onzalo \\ Ravesta, María Inés Pino, G onzalo Valdivia², Manuel \\ Moreno $^{1}$, Luis Villarroel $2, b$. \\ Influence of obesity on health care \\ costs and absenteeism among \\ employees of a mining company
}

\begin{abstract}
Background: The bealth associated costs of obesity can represent between 2\% and 9\% of the total bealth costs of a given country. Aim: To assess the impact of obesity on bealth care costs and absenteeism in a cohort of mine workers. Patients and methods: Prospective study of 4.673 men, employees of a mining company, aged $49 \pm 7$ years that were followed for $24 \pm 11$ months. Total health care cost and days of sick leave were recorded for each individual. The association between obesity and these variables was analyzed by logistic regression adjusting for co-morbidities, age and other variables. Results: Mean annual health care costs for obese workers were 17\% higher ( $p<0.001)$ compared to workers with normal weight and 58\% higher $(p<0.001)$ for workers with severe and morbid obesity. Mean annual days of sick leave increased by 25\% in the obese $(p=0.002)$ and by $57 \%$ in subjects with severe and morbid obesity $(p<0.001)$. For health care costs the most significant predictors were: presence of diabetes mellitus (Odds ratio (OR) 6.21, 95\% confidence intervals (95\% CI) 4.9 to 7.9), bypertension (OR 3.99; 95\% CI 3.4 to 4.6) and severe and morbid obesity (OR 2.55, 95\% CI 1.9 to 3.4). For absenteeism the most significant predictors were: presence of diabetes mellitus (OR 1.58, 95\% CI 1.2 to 2.0), bypertension (OR 1,34, 95\% CI 1.2 to 1.6) and severe and morbid obesity (OR 1.50, 95\% CI 1.1 to 2.1). Conclusions: Obesity increases significantly health care costs and absenteeism (Rev Méd Chile 2009; 137: 337-44).
\end{abstract}

(Key words: Comorbidity; Health expenditures; Obesity)

\begin{abstract}
angeniero ejecución químico
${ }^{\mathrm{b}}$ Doctor en estadística ni preparación del manuscrito.
\end{abstract}

Recibido el 14 de agosto, 2008. Aceptado el 5 de diciembre, 2008.

${ }^{1}$ Departamento de Nutrición, Diabetes y Metabolismo y ${ }^{2}$ Departamento de Salud Pública, Facultad de Medicina, Pontificia Universidad Católica de Chile. Santiago, Chile.

Este trabajo se realizó con la autorización de CODELCO, institución a la que pertenecen algunos autores, pero no tuvo ingerencia en el diseño del estudio, el análisis de resultados

Correspondencia a: Dr. Aldo Zárate. Departamento de Nutrición, Diabetes y Metabolismo, Facultad de Medicina, Pontificia Universidad Católica de Chile. Lira 40. Santiago, Chile. Fono: (56-2) 6863862. Fax: (56-2) 6338298.

E mail: zarate.aldo@gmail.com 
$\mathrm{L}$ a obesidad es reconocida como un importante problema de salud pública en todo el mundo. El aumento de su prevalencia ha alcanzado caracteres epidémicos para la gran mayoría de los países industrializados y en desarrollo ${ }^{1}$. En Chile, en un estudio en población urbana en 1997, se encontró una prevalencia de 19,7\% y la Encuesta Nacional de Salud del año 2003, con representatividad nacional, mostró una prevalencia de $23,2 \%{ }^{2,3}$.

Según los estudios publicados, el costo que demanda la obesidad y sus comorbilidades asociadas, alcanza un porcentaje que varía entre $2 \%$ y $9,4 \%$ del costo total en salud en países con información disponible ${ }^{4,5}$. En los Estados Unidos de Norteamérica, desde el año 1995 hasta 2003, el costo anual relacionado con la obesidad derivada del uso de servicios de salud (costo directo), aumentó de U\$ 51,6 a U\$ 75 billones ${ }^{6,7}$. Al comparar el costo directo anual entre sujetos con peso normal con sujetos obesos, los rangos de aumento porcentual oscilan entre 25\% y 36\% más alto para obesos y entre $44 \%$ y $53 \%$ mayor en el caso de obesidad severa y mórbida ${ }^{8-10}$.

Son pocos los estudios de costos relacionados con la pérdida de productividad asociada a la obesidad (costos indirectos). La mayor parte de ellos se centran en la determinación de ausentismo laboral y los resultados son dispares dependiendo de la metodología empleada, variando desde la ausencia de asociación hasta un aumento en el doble o más de días de licencia médica11-14.

La mayoría de los análisis de costos de la obesidad corresponden a estimaciones derivadas de estudios transversales, basadas en el riesgo atribuible poblacional ${ }^{4}$. De esta forma, la cuantificación de los costos de la obesidad se hace valorizando enfermedades, para las cuales se asume que la obesidad juega un rol etiológico relevante en una proporción preestablecida. Esta metodología conlleva riesgo intrínseco de sesgo, ya que cada estudio define por arbitrio del investigador, un grupo de enfermedades en las que, en mayor o menor medida, la obesidad satisface dicho rol etiológico. En cambio, los estudios que utilizan datos individuales de pacientes, que son aún más escasos, permiten una aproximación más real del costo adicional asociado a la obesidad, permitiendo el ajuste por variables demográficas, médicas y laborales relevantes.
A nivel nacional, el impacto económico del sobrepeso y de la obesidad no ha sido evaluado y tampoco se dispone de datos sobre el ausentismo laboral de causa médica atribuible al exceso de peso.

El objetivo de este trabajo es determinar el costo en salud y el ausentismo laboral asociado a la obesidad, utilizando datos a nivel individual, evaluando también el impacto producido por la presencia de algunas comorbilidades asociadas (diabetes, hipertensión arterial y dislipidemias) con el fin de respaldar la implementación de un programa de prevención, pesquisa y tratamiento de la obesidad, el cual irá en beneficio directo de la población trabajadora.

\section{MATERIAL y MÉTOdOS}

Se utilizó un registro prospectivo de 4.673 trabajadores de una empresa minera incorporados al estudio tras la realización obligatoria de su examen de salud laboral, entre el 01/01/2003 y el 31/ $12 / 2006$. Los sujetos fueron seguidos consignando el uso del Servicio de Salud local y el ausentismo laboral desde el 01/01/2004 hasta el 30/06/2007. De esta manera el periodo máximo de seguimiento fue de 42 meses y el mínimo de 6 meses. Durante el seguimiento no se registraron egresos, fallecimientos o pérdidas de información de ningún trabajador de la cohorte.

Población objetivo. Los criterios de ingreso fueron: trabajador activo; sexo masculino; ingreso a la empresa antes del 01/01/2004.

Datos de uso del Servicio de Salud. Se obtuvieron a través de la ficha clínica informatizada utilizada por el Servicio Médico a cargo de la población trabajadora. En ella se registran las siguientes prestaciones: número de consultas de cada sujeto, el listado específico de exámenes de laboratorio, de exploraciones complementarias (imagenología, procedimientos diagnósticos asociados a especialidades y exámenes de anatomía patológica) y de los fármacos prescritos (descripción y cantidad). Tanto en laboratorio, exploraciones complementarias y fármacos el listado comprende el total de órdenes emanadas en atención ambulatoria y hospitalizada. 
Se excluyen de este registro las intervenciones quirúrgicas, los días-cama derivados de hospitalización y la hemodiálisis, por no disponer de antecedentes completos de esas prestaciones. De esta manera el registro del uso de Servicio de Salud cubre totalmente la atención ambulatoria y en forma parcial la atención hospitalizada.

Datos de costo en salud. El costo del uso del Servicio de Salud se definió como la suma de los costos de las prestaciones que lo componen. Para la valorización del costo de consultas, exámenes de laboratorio y complementarios, se fijó el costo en 100\% del arancel FONASA (nivel 1) multiplicado por un factor fijo de 1,6, el cual corresponde a un ajuste tarifario local. Se excluyeron por tanto, prestaciones para las cuales no se dispone del correspondiente código FONASA. Para el ítem farmacia, el costo calculado fue equivalente al costo real del fármaco según el precio de licitación, considerando el valor del dólar en $\$ 530$ pesos (marzo 2006).

Antecedentes laborales. El número y la duración de las licencias médicas de cada trabajador se obtuvieron a través del registro informatizado de asistencia aportado por la empresa. No se tuvo acceso a los diagnósticos ligados a cada licencia médica.

Estado nutricional. Los sujetos fueron clasificados según el Índice de Masa Corporal (IMC). El peso y la estatura fueron determinados durante el examen de salud laboral por una enfermera entrenada, empleando una balanza marca Seca con tallímetro, modelo 713. Los trabajadores fueron clasificados en su estado nutricional de acuerdo a lo sugerido por la Organización Mundial de la Salud (OMS): IMC de 18,5 a $24,9 \mathrm{Kg} / \mathrm{m}^{2}=$ normales; IMC entre 25 y 29,9 =sobrepeso; de 30 a 34,9 =obesidad y con IMC de 35 o más =obesidad severa y mórbida.

Comorbilidades. La presencia de hipertensión arterial (HTA), diabetes mellitus (DM) y dislipidemia (DLP), se identificó usando el registro del Programa de Riesgo Cardiovascular del Servicio de Salud local, estableciendo el diagnóstico según los criterios de normas ministeriales vigentes ${ }^{15,16}$. El tabaquismo se determinó a través de un cuestionario realizado en el examen de salud laboral.
Variables dependientes. Se analizaron por separado el promedio del costo anual en salud (valorizado en pesos) y el promedio de días de licencia médica anual para cada trabajador. Para el análisis univariado se usó la transformación logarítmica de ambas variables, dada la naturaleza asimétrica de su distribución. Los resultados se presentan en la escala original. Para el análisis de regresión se fijó el percentil 75 de ambas, a fin de identificar a los trabajadores que demandaron mayores costos y licencias médicas.

Variables independientes. Se consideraron la edad, el estado civil, la antigüedad en la empresa, el IMC, el estado nutricional (IMC), el nivel de remuneraciones, tipo de área laboral, tipo de cargo y la presencia de HTA, DM, DLP y tabaquismo. Las variables continuas fueron tratadas como continuas o categóricas, según tipo de análisis, confeccionando variables binarias.

Estadística. Se utilizó el programa SPSS v.12 para ANOVA y chi cuadrado con un nivel de significación de 5\%. Los datos se presentan como promedios y desviación estándar (DE). Se determinaron los "odds ratios" (OR) con intervalos de confianza al 95\% (IC 95\%), a través de una regresión logística utilizando el percentil 75 de cada variable dependiente. Se fijó este percentil de acuerdo a referencias previas ${ }^{11,17}$. Las variables independientes HTA, DM y DLP fueron incluidas en el modelo de regresión logística considerando evidencias que demuestran que el efecto del IMC sobre costos, salud o mortalidad $^{18-21}$ es atenuado, pero no eliminado tras ajustar por factores de riesgo potencialmente intermediarios, dando lugar a un efecto independiente del IMC.

Los criterios de entrada y salida para las variables independientes fueron 0,05 y 0,1 , respectivamente y se usó la eliminación posterior ("backward") basada en el "likelihood ratio". La bondad de ajuste del modelo se analizó con la prueba de Hosmer-Lemeshow.

\section{Resultados}

Análisis univariado. El rango de edad de la cohorte fue de 27 a 74 años, promedio 49,2 años. 
El seguimiento promedio de la cohorte fue de 23,9 meses, sin diferencias significativas entre las categorías de IMC. La prevalencia de sobrepeso, obesidad y obesidad severa y mórbida fue 56,2\%; 24,3\% y 3,9\%, respectivamente. Los trabajadores con mayor IMC presentaron mayor prevalencia de HTA, DM y DLP y mayor promedio de edad. Se encontró una relación inversa entre prevalencia de tabaquismo e IMC (Tabla 1).

Las distribuciones del costo en salud y de ausentismo laboral resultaron ser asimétricas. El costo anual promedio en salud fue de $\$ 237.174$ (mediana \$165.638) y el promedio anual de ausentismo laboral fue de 14,1 días (mediana 5,4 días). Los costos en salud aumentaron desde $\$ 217.270$, para el estado nutricional normal a $\$ 343.028$ en sujetos obesos severos y mórbidos ( $\mathrm{p}<0,001)$. El promedio de días de licencia médica aumentan desde 12,6 días en el estado nutricional normal a 19,9 días en obesos severos y mórbidos ( $p<0,001$ ) (Tabla 2). Aunque estadísticamente significativa $(\mathrm{p}<0,001)$ la correlación lineal entre IMC versus costos en salud $\mathrm{y}$ ausentismo es difícil de interpretar $\left(\mathrm{r}^{+}{ }^{+} 0,128 \mathrm{y}{ }^{+} 0,08\right.$, respectivamente).

La frecuencia de trabajadores que incurrieron en un costo cero en salud fue inferior a 0,1\%. En cambio, $13 \%$ de los trabajadores no registraron licencia médica durante el seguimiento (652 de
4.673). El costo en salud se distribuyó en 29,9\% en consultas médicas, 15,8\% en exámenes de laboratorio, $19,6 \%$ en exploraciones complementarias, correspondiendo 34,7\% al ítem de farmacia.

Según categorías de edad, los costos en salud se incrementan desde $\$ 164.411$ en sujetos de 20 a 39 años a \$305.418 en los 55 o más años ( $p<0,001)$. Se observó una tendencia al incremento de los costos en salud conforme aumenta la edad y el estado nutricional (Tabla 3). En ausentismo laboral, no se observó diferencia significativa entre las diferentes categorías de edad, con 14,6 días en los trabajadores de 20 a 39 años y de 13,6 días para los de 55 o más años.

Las comorbilidades asociadas a la obesidad (HTA, DM y DLP) incidieron en un aumento del costo en salud en 77\%, 130\% y 48\%, respectivamente ( $\mathrm{p}<0,001)$ y en 37\%, 57\% y 17\% de aumento en el ausentismo laboral ( $p<0,001)$. Por el contrario, el tabaquismo disminuyó el costo en salud en 11\% ( $\mathrm{p}=0,006)$ y aumentó los días de licencia médica en 19\% ( $\mathrm{p}<0,001$ ) (Tabla 4). El grupo de fumadores tuvo significativamente menor edad (47,7 años; p <0,001) y menor prevalencia de obesidad, HTA y DM (26\%, 15,8\% y 5,4\%, respectivamente; $\mathrm{p}<0,001)$ que los no fumadores.

En relación a las variables de índole laboral, sólo el nivel de remuneraciones (agrupadas en 4 catego-

Tabla 1. Características de la cohorte de trabajadores al ingreso al estudio, según estado nutricional.

\begin{tabular}{|c|c|c|c|c|c|c|}
\hline & \multicolumn{6}{|c|}{ Estado nutricional } \\
\hline & Total & Normal & Sobrepeso & Obeso & $\begin{array}{c}\text { Obeso } \\
\text { severo y mórbido }\end{array}$ & $\mathbf{p}^{1}$ \\
\hline n (\%) & $4.673(100)$ & $720(15,4)$ & $2.630(56,2)$ & $1.138(24,3)$ & $185(3,9)$ & \\
\hline Edad (años) ${ }^{2}$ & $49,2 \pm 7,4$ & $48,4 \pm 8,1$ & $49,2 \pm 7,4$ & $49,3 \pm 7,2$ & $50,2 \pm 6,4$ & 0,01 \\
\hline Antigüedad (años) ${ }^{2}$ & $23,8 \pm 8,0$ & $22,8 \pm 8,6$ & $23,8 \pm-8,0$ & $24,0 \pm 7,8$ & $25,7 \pm 7,0$ & $<0,001$ \\
\hline Peso $(\mathrm{kg})^{2}$ & $81,2 \pm 11,8$ & & & & & \\
\hline Talla $(\mathrm{m})^{2}$ & $1,69 \pm 0,06$ & & & & & \\
\hline IMC $\left(\mathrm{kg} / \mathrm{m}^{2}\right)^{2}$ & $28,3 \pm 3,5$ & & & & & \\
\hline Tabaco (\%) & 39,6 & 46,7 & 39,4 & 36,1 & 35,6 & $<0,001$ \\
\hline HTA (\%) & 23,0 & 14,0 & 19,9 & 31,0 & 53,0 & $<0,001$ \\
\hline DM (\%) & 6,9 & 5,4 & 5,6 & 9,0 & 17,3 & $<0,001$ \\
\hline DLP (\%) & 23,1 & 14,9 & 21,5 & 29,5 & 36,2 & $<0,001$ \\
\hline Seguimiento (meses) ${ }^{2}$ & $23,9 \pm 10,7$ & $24,0 \pm 10,7$ & $24,1 \pm 10,8$ & $23,5 \pm 10,5$ & $23,3 \pm 11,0$ & NS \\
\hline
\end{tabular}

${ }^{1}$ p por ANOVA para edad y antigüedad y $x^{2}$ para el resto de las variables. ${ }^{2}$ promedio \pm desviación estándar. HTA: hipertensión arterial; DM: diabetes mellitus; DLP: dislipidemia 
Tabla 2. Costos en salud y ausentismo laboral anuales según estado nutricional. (Pesos promedio \pm desviación estándar)

\begin{tabular}{|c|c|c|c|c|c|c|}
\hline & \multirow[b]{2}{*}{ Total } & \multirow[b]{2}{*}{ Normal } & \multicolumn{2}{|c|}{ Estado nutricional } & \multirow[b]{2}{*}{$\begin{array}{c}\text { Obeso } \\
\text { severo y mórbido }\end{array}$} & \multirow[b]{2}{*}{$\mathrm{p}^{1}$} \\
\hline & & & Sobrepeso & Obeso & & \\
\hline $\mathrm{n}(\%)$ & $4.673(100)$ & $720(15,4)$ & $2.630(36,2)$ & $1.138(24,3)$ & $185(3,9)$ & \\
\hline Costo en salud (pesos) & $237.174,8 \pm 285.972,2$ & $217.270,4 \pm 305.323,1$ & $227.462,4 \pm 282.918,5$ & $255.005,7 \pm 271.087$ & $343.028,9 \pm 313.576,5$ & $<0,001$ \\
\hline Ausentismo laboral (días) & $14,1 \pm 24,3$ & $12,6 \pm 22,9$ & $13,3 \pm 23,2$ & $15,8 \pm 26,6$ & $19,9 \pm 27,8$ & $<0,001$ \\
\hline
\end{tabular}

${ }^{1}$ p por ANOVA para Log (costo en salud) y Log (ausentismo laboral).

Tabla 3. Costo en salud anual según estado nutricional y rango de edad. (Pesos promedio \pm desviación estándar)

\begin{tabular}{|c|c|c|c|c|c|c|}
\hline \multirow[b]{2}{*}{$\begin{array}{l}\text { Rango de edad } \\
\text { (años) }\end{array}$} & \multirow[b]{2}{*}{ Total } & \multirow[b]{2}{*}{ Normal } & \multicolumn{2}{|c|}{ Estado nutricional } & \multirow[b]{2}{*}{$\begin{array}{c}\text { Obeso } \\
\text { severo y mórbido }\end{array}$} & \multirow[b]{2}{*}{$\mathbf{p}^{1}$} \\
\hline & & & Sobrepeso & Obeso & & \\
\hline 20 a 39 años & $164.411 \pm 138.658$ & 141.928 & $154.616 \pm 1$ & $186.711^{*}$ & 176.949 & 0,002 \\
\hline 40 a 44 años & $197.229 \pm 189.348$ & $187.482=$ & $186.958 \pm 179.727$ & $211.794 \pm 191.832$ & $296.347 \pm 306.154^{*}$ & 0,005 \\
\hline 45 a 49 años & $224.780 \pm 316.621$ & $215.678 \pm 406.890$ & $211.549 \pm 326.454$ & $249.756 \pm 233.003$ & $289.720 \pm 185.495$ & NS \\
\hline 50 a 54 años & $236.203 \pm 239.781$ & $206.811 \pm 196.936$ & $234.181 \pm 246.511$ & $244.891 \pm 252.109$ & $291.765 \pm 181.806$ & NS \\
\hline 55 años o más & $305.418 \pm 370.079$ & $290.009 \pm 382.822$ & $290.824 \pm 355.941$ & $316.956 \pm 371.030$ & $493.769 \pm 455.504^{*}$ & 0,002 \\
\hline
\end{tabular}

${ }^{1}$ p por ANOVA para Log (costo en salud) según rango de edad e IMC

*Diferencia significativa en test de comparaciones múltiples

Tabla 4. C ostos en salud y ausentismo laboral anuales según comorbilidades (promedio \pm desviación estándar)

\begin{tabular}{|lcccrrrr|}
\hline & & \multicolumn{2}{c}{ Costo en salud (pesos) } & \multicolumn{4}{c|}{ Ausentismo laboral (días) } \\
$\begin{array}{l}\text { Presencia de } \\
\text { comorbilidad }\end{array}$ & Sín & No & $\mathbf{p}^{\mathbf{1}}$ & Sí & No & $\mathbf{p}^{\mathbf{1}}$ \\
\hline Hipertensión arterial & $356.685 \pm 374.253$ & $201.468 \pm 242.602$ & $<0,001$ & $17,7 \pm 29,6$ & $12,9 \pm 22,4$ & $<0,001$ \\
Diabetes Mellitus & $501.227 \pm 488.936$ & $217.699 \pm 254.378$ & $<0,001$ & $21,2 \pm 33,7$ & $13,5 \pm 23,4$ & $<0,001$ \\
Dislipidemia & $315.995 \pm 306.465$ & $213.454 \pm 275.170$ & $<0,001$ & $15,8 \pm 26,7$ & $13,5 \pm 23,5$ & 0,006 \\
Tabaquismo & $217.069 \pm 246.116$ & $242.856 \pm 313.619$ & 0,002 & $14,8 \pm 22,5$ & $12,4 \pm 21,3$ & $<0,001$ \\
\hline
\end{tabular}

${ }^{1}$ p por ANOVA para Log (costo en salud) y Log (ausentismo laboral)

rías) incidió significativamente en el ausentismo laboral. En la categoría de remuneración más baja hubo 19,5 días, mientras en la más alta 8,3 días ( $\mathrm{p}<0,001$ ).

Análisis multivariado. Para el costo en salud, las variables seleccionadas por el modelo utilizado fueron: estado nutricional según IMC, rango de edad, nivel de remuneración, hipertensión arterial, diabetes y dislipidemia (Tabla 5). La asociación entre obesidad severa y mórbida y alto costo en salud (OR 2,82; IC 95\% 2,09-3,81; p <0,001) se mantuvo aun al ajustar por estas variables (OR 
Tabla 5. 0 dds ratio (OR) e intervalos de confianza al $95 \%$ (95\% IC) para variables seleccionadas según costos en salud y ausentismo laboral

\begin{tabular}{|c|c|c|c|c|}
\hline & \multicolumn{2}{|c|}{ Costo en salud } & \multicolumn{2}{|c|}{ Ausentismo laboral } \\
\hline & OR $(95 \%$ IC $)$ & $\begin{array}{c}\text { OR ajustado } \\
\text { (95\% IC })^{*}\end{array}$ & OR $(95 \%$ IC) & $\begin{array}{l}\text { OR ajustado } \\
(95 \% \text { IC })^{* *}\end{array}$ \\
\hline Hipertensión arterial & $3,98(3,44-4,61)$ & $3,0(2,55-3,53)$ & $1,34(1,15-1,56)$ & $1,39(1,19-1,63)$ \\
\hline Diabetes mellitus & $6,21(4,89-7,89)$ & $4,22(3,26-5,47)$ & $1,57(1,23-2,00)$ & $1,57(1,22-2,02)$ \\
\hline Dislipidemia & $2,49(2,15-2,89)$ & $1,44(1,22-1,70)$ & $1,18(1,01-1,38)$ & NS \\
\hline Obesidad severa y mórbida & $2,82(2,09-3,81)$ & $1,65(1,18-2,30)$ & $1,58(1,15-2,17)$ & NS \\
\hline Rango de edad 20 a 39 años. & $0,23(0,16-0,32)$ & $0,38(0,27-0,55)$ & NS & NS \\
\hline Categoría de remuneración más alta & NS & NS & $0,35(0,28-0,43)$ & $0,32(0,25-0,40)$ \\
\hline
\end{tabular}

*Variables en el modelo: estado nutricional, categorías de edad, nivel de remuneración, presencia de hipertensión, diabetes y dislipidemia $\left(\mathrm{R}^{2}=0,178\right.$; Test Hosmer-Lemeshow, $\left.\mathrm{p}=0,955\right)$.

**Variables en el modelo: estado nutricional, categorías de edad, nivel de remuneración, presencia de hipertensión, diabetes y dislipidemia $\left(\mathrm{R}^{2}=0,04\right.$, Test Hosmer-Lemeshow, $\mathrm{p}=0$,616).

NS: no significativo

1,65; IC 95\% 1,18-2,30); $\mathrm{p}=0,003)$. Al analizar las comorbilidades, la presencia de hipertensión (OR 3,98; IC 95\% 3,44-4,61; p <0,001) explica la mayor parte del potencial predictivo del modelo (pseudo $\left.\mathrm{R}^{2}=0,178\right)$, siendo también significativa la influencia de diabetes, dislipidemia y el rango de edad de 20 a 39 años.

Para el ausentismo laboral, el modelo final incluyó las siguientes variables: estado nutricional según IMC, rango de edad, nivel de remuneración, presencia de hipertensión y de diabetes. La asociación entre obesidad severa y mórbida con ausentismo laboral (OR 1,58; IC 95\% 1,15-2,17; p $=0,005$ ) se pierde, al ajustar por el conjunto de potenciales variables confundentes (Tabla 5). Al igual que el caso anterior, el predictor más relevante fue la presencia de hipertensión (OR 1,34; IC 95\% 1,15-1,56; p <0,001), manteniéndose significativas además la presencia de diabetes y la categoría de remuneración más alta.

\section{DisCUSIÓN}

Este estudio permitió determinar el impacto del exceso de peso en cuanto a costos en salud y ausentismo laboral en una cohorte de trabajadores de una compañía minera en Chile. Si bien es una población seleccionada, que incluye sólo hombres adultos, tiene la ventaja de permitir un análisis basado en rigurosos registros individuales de las prestaciones médicas otorgadas en el periodo definido, sin pérdida de información. La prevalencia de obesidad encontrada fue de $28,2 \%$, más alta que la descrita en la Encuesta Nacional de Salud a nivel nacional para hombres de edades similares $(23,6 \%)^{2}$.

Los resultados confirman la relación descrita entre obesidad y, especialmente, de la obesidad severa y mórbida, con el incremento del costo en salud, que resultó ser mayor en 17\% y 58\%, respectivamente, al observado en los sujetos con IMC normal (Tabla 2). Estas cifras, que concuerdan con las descritas en la literatura ${ }^{8-14}$, comprenden todos los componentes del costo en salud considerados en este estudio: consultas médicas, exámenes de laboratorio y de farmacia (datos no mostrados).

Debido a la forma con la que se definió el costo en salud en esta cohorte de trabajadores, ellos son sólo parcialmente comparables a los informados por la Superintendencia de Salud de Chile para el sector público y privado. Las principales diferencias subyacen en la exclusión de prestaciones hospitalarias, como intervenciones quirúrgicas, días-cama y otras acciones terapéuticas de mayor complejidad, que inciden en el costo de salud informado. De hecho, para el 
sistema de Instituciones de Salud Privadas (ISAPRES) en el año 2006, aun cuando las atenciones de tipo hospitalario sólo representaron $11 \%$ del total de las prestaciones médicas otorgadas, correspondieron a $42 \%$ del total de los montos facturados ${ }^{22}$. En este sentido, los costos en salud aquí presentados subestiman el costo real. Otra diferencia notoria ocurre con la valorización de los medicamentos. En la población estudiada, el Servicio de Salud local cubre la totalidad de los costos en pacientes ambulatorios y hospitalizados, a diferencia de lo que ocurre en el sistema público y privado en los cuales la única aproximación real al gasto en fármacos se ha realizado en estudios de "gasto-de-bolsillo" en salud por quintiles de ingreso familiar ${ }^{23}$.

Los costos en salud aumentan notoriamente con la presencia de comorbilidades de alta prevalencia asociadas a la obesidad (Tablas 1 y 4 ). Sin embargo, cuando se analiza el alto costo en salud determinado por el percentil 75 , luego del ajuste por variables seleccionadas incluyendo hipertensión, diabetes y dislipidemia, la obesidad severa y mórbida mantiene su efecto significativo (OR de $1,65)$, demostrando ser un factor independiente (Tabla 5).

En cuanto al ausentismo laboral, son escasos los estudios publicados en Chile y más específicamente, en compañías mineras. En la minera El Indio, con una población similar a este estudio (4.575 trabajadores), el promedio de días de licencia médica anuales en el año 1993 fue de 13,8 días. En la División Andina de Codelco, entre los años 1989 y 2001, el promedio fluctuó entre 8,1 y 10 días de licencia médica anual ${ }^{24}$.

Los resultados de este estudio muestran que el ausentismo laboral aumenta notoriamente según

\section{REFERENCIAS}

1. World Health Organization. $37^{\text {th }}$ Session of the Subcommittee on Planning and Programming of the Executive Committee: Obesity, Diet, and Physical Activity. 2003; Consultado el 3/11/2008 en: www.paho.org/English/GOV/CE/SPP/spp37-08-e.pdf.

2. MINSAL. Encuesta Nacional de Salud 2003. Consultado el 3/11/2008 en: http://epi.minsal.cl/epi/html/ invest/ENS/InformeFinalENS.pdf.

3. Jadue L, Vega J, Escobar MC, Delgado I, Garrido C, LASTRA P ET AL. Factores de riesgo para las enfermeda- aumenta la categoría nutricional determinada por IMC. Existe un incremento neto en los sujetos obesos y en los obesos severos y mórbidos de $25 \%$ y $57 \%$, respectivamente (Tabla 2 ). La influencia de las morbilidades es similar al incremento observado en los costos en salud (Tabla 4). Finalmente, a pesar de que existe asociación estadística entre estado nutricional y alto ausentismo laboral, el potencial predictivo escaso del modelo (pseudo $\mathrm{R}^{2}=0,04$ ) sugiere la existencia de variables no incluidas en este modelo que pueden incidir en forma más significativa.

Con respecto al tabaquismo, los resultados son dispares. El menor costo en salud probablemente se explique por la menor edad de los fumadores y la menor prevalencia de comorbilidades observada en este grupo de trabajadores. En cuanto al ausentismo laboral, el efecto observado es similar a lo informado en la literatura como efecto independiente del tabaco ajustado por la condición de salud ${ }^{25}$.

En conclusión, los resultados confirman que la obesidad, especialmente la severa y mórbida, tiene un efecto muy significativo en el aumento en los costos en salud y en el ausentismo laboral por efecto directo y asociado a las comorbilidades que la acompañan. Con estos antecedentes, las medidas de prevención y tratamiento de la obesidad pueden ser analizadas en sus relaciones costoefectividad y costo-beneficio para mejorar la salud de las personas y su productividad laboral.

\section{Agradecimientos}

Al Programa Magíster en Nutrición Clínica del Departamento de Nutrición, Diabetes y Metabolismo de la Pontificia Universidad Católica de Chile por su orientación en la génesis, la evolución y conclusiones del estudio.

des no transmisibles: Metodología y resultados globales de la encuesta de base del programa CARMEN (Conjunto de Acciones para la Reducción Multifactorial de las Enfermedades no Transmisibles). Rev Méd Chile 1999; 127: 1004-13.

4. Thompson D, Wolf AM. The medical-care cost burden of obesity. Obes Rev 2001; 2: 189-97.

5. Coldtiz GA. Economic costs of obesity and inactivity. Med E Sci Sports Exerc 1999; 31: S663-S667.

6. Wolf AM, Colditz GA. Current estimates of the economic cost of obesity in the United States. Obes Res 1998; 6: 97-106. 
7. State-level estimates of annual medical expenditures attributable to obesity. Obes Res 2004; 12: 18-24.

8. Quesenberry CP Jr, CaAn B, Jacobson A. Obesity, health services use, and health care costs among members of a health maintenance organization. Arch Intern Med 1998; 158: 466-72.

9. Thompson D, Brown JB, Nichols GA, Elmer PJ, Oster G. Body mass index and future health-care costs: A retrospective cohort study. Obes Res 2001; 9: 210-8.

10. Anderson lH, Martinson BC, Crain AL, Pronk NP, Whitebird RR, O'Connor PJ, FIne LJ. Health care charges associated with physical inactivity, overweight, and obesity. Prev Chronic Dis 2005; 2: A09. Epub 2005 Sep 15.

11. Moreau M, Valente F, Mak R, Pelfrene E, De Smet P, De BaCKer G, Kornitzer M. Obesity, body fat distribution and incidence of sick leave in the Belgian workforce: the Belstress study. Int J Obes Relat Metab Disord 2004; 28: 574-82.

12. Burton WN, Chen C-Y, Schultz AB, Edington DW. The economic costs associated with body mass index in a workplace. J Occup Environ Med 1998; 40: 786-92.

13. Narbro K, Jonsson E, Larsson B, Waaler H, Wedel H, Sjostrom L. Economic consequences of sick-leave and early retirement in obese Swedish women. Int J Obes 1996; 20: 895-903.

14. Tsai SP, Ahmed FS, Wendt JK, Bhojani F, Donnelly RP. The impact of obesity on illness absence and productivity in an industrial population of petrochemical workers. Ann Epidemiol 2008; 18: 8-14. Epub 2007 Sep 24.

15. MINSAL. Programa de Salud Cardiovascular. Reorientación de los programas de hipertensión y diabetes. Consultado el 3/11/2008 en: http:// webhosting.redsalud.gov.cl / minsal/ archivos/saludcardiovascular/Reorientación de los Programas de Hipertensión y Diabetes. pdf..
16. MINSAL. Programa de Salud Cardiovascular. Dislipidemias. Consultado el 3/11/2008 en: http:// webhosting.redsalud.gov.cl/minsal/archivos/saludcardiovascular/guia_dislipidemias.pdf.

17. Bungum T, Satterwhite M, Jackson AW, Morrow JR. The relationship of body mass index, medical costs, and job absenteeism. Am J Health Behav 2003; 27: 456-62.

18. Hubert HB, Feinlieb M, McNamara PM, Cateslli WP. Obesity as an independent risk factor for cardiovascular disease: a 26-year follow-up of participants in the Framingham Heart Study. Circulation 1983; 67: 968-77.

19. Daviglus ML, Liu K, Yan Ll, Pirzada A, Manheim L, ManNing W ET AL. Relation of body mass index in young adulthood and middle age to Medicare expenditures in older age. JAMA 2004; 292: 2743-9.

20. Pronk NP, Goodman MJ, O'Connor PJ, Martinson BC. Relationship between modifiable health risks and short-term health care charges. JAMA 1999; 282: 2235-9.

21. Dorn JM, Schisterman EF, Winkelstein W Jr, Trevisan M. Body mass index and mortality in a general population sample of men and women. The Buffalo Health Study. Am J Epidemiol 1997; 146: 919-31.

22. Superintendencia de Salud. Departamento de Estudios y Desarrollo. Boletin Estadístico 2006. Consultado el 3/11/2008 en: /569/" http://www.supersalud.cl/ documentacion /569/ articles-3584_recurso_1.pdf.

23. MINSAL. Primer Estudio Nacional sobre Satisfacción y Gasto en Salud. Informe de Resultados. Módulos "F" $y$ "G" Gasto de Bolsillo en Salud. 2006. Consultado el 3/11/2008 en: http://163.247.51.38/desarrollo/css/desal/ENSGS_Gasto.pdf.

24. Mesa FR, KaempfFer AM. 30 años de estudio sobre ausentismo laboral en Chile: una perspectiva por tipos de empresas. Rev Méd Chile 2004; 132: 1100-8.

25. Lundborg P. Does smoking increase sick leave? Evidence using register data on Swedish workers. Tob Control 2007; 16: 114-8. 